\title{
Evaluating a designated family planning clinic within a genitourinary medicine clinic
}

\author{
E M Carlin, J M Russell, K Sibley, F C Boag
}

\begin{abstract}
Objective-To evaluate an integrated family planning clinic (FPC) established by genitourinary medicine (GUM) staff held within a GUM women-only clinic (WOC).
\end{abstract}

Design-A retrospective case note review of women attending the FPC during the first year January-December 1992.

Results-One hundred and thirteen women, aged 13-41 years, attended the FPC; 45 were new attenders, six had previously tested antibody positive for the human immunodeficiency virus (HIV), seven were intravenous drug users; $54 \%$ had a history of sexually transmitted disease (STD); $17 \cdot 7 \%$ were using no contraception; $32 \cdot 7 \%$ had previous termination of pregnancy (TOP) with 70 TOPs in total. Within three months of FPC attendance $89(78 \cdot 8 \%)$ women had genital STD screening performed; syphilis, HIV and hepatitis $B$ serology, together with cervical cytology were performed in $77,18,13$ and 62 women respectively. Infections identified were similar to those identified in the GUM clinic but the prevalence of Chlamydia trachomatis in diagnosed infections was commoner in FPC attenders and epidemiological treatment commoner in GUM attenders. No high grade cytology abnormalities were detected. No positive syphilis or new HIV positive results were identified; five women were found to be hepatitis $B$ surface antibody positive. Contraception was changed in $60 \cdot 8 \%$. Most frequently supplied was the combined oral contraceptive pill (COCP). At the first FPC attendance six women required post coital contraception (PCC) and five were already pregnant, three suspected it, two were unaware. During the year three women conceived; two used COCP, but were non compliant; one used a diaphragm with unclear compliance. Seven of the eight pregnancies were terminated. Over the following year, 1992-93, contraception was supplied to 42 women; four required PCC; two intentional pregnancies occurred. Only one of the TOP women returned.

Conclusion-An integrated FPC provides co-ordinated sexual health care. Pregnancy, TOP and FPC re-attendance rates together with improvement strategies are discussed. Avoiding unwanted pregnancy remains a universal challenge.

(Genitourin Med 1995;71:106-108)
Keywords: Family planning; Genitourinary medicine clinics

Introduction

The reduction of unwanted pregnancies and sexually transmitted diseases (STDs) have been identified as target areas for improving sexual health in the government White Paper The Health of the Nation.

Currently, family planning and genitourinary medicine (GUM) services are not closely linked. However, women attending a family planning clinic (FPC) are usually sexually active and in one study $53 \%$ of women self reported one or more prior episodes of STD. The prevalence of specific STDs in FPC attenders varies but has been reported as 5-13\% for Chlamydia trachomatis, ${ }^{3-6}$ 4-11\% for Trichomonas vaginalis ${ }^{78}$ and $0-2 \%$ for Neisseria gonorrhoeae. ${ }^{78}$ The prevalence of infection with the human immunodeficiency virus (HIV) in United Kingdom FPC attenders is not known but in Philadelphia serum antibodies to HIV type 1 have been identified in $1.1 \%$ of FPC attenders. ${ }^{9}$

Looking at GUM clinic attenders, a significant number of women are at risk of unwanted pregnancy either because of unreliable or absent contraception use. In 1991 $40 \%$ of women attending our own GUM clinic, the John Hunter Clinic (JHC), were at such risk compared with $21 \%$ and $26 \%$ of women attending the other two GUM clinics in the Riverside Health District. ${ }^{10}$ This differential may have been related to reduced FPC access as, at this time, the JHC was isolated from a general hospital whereas at the two other sites there was either an integral FPC or easy access to family planning services. Queen and colleagues have also noted that women are more likely to be at risk if aged under 20 years or if not registered with a general practitioner. ${ }^{11}$

In 1991, in response to womens' requests, a women-only clinic (WOC) staffed exclusively by and for women was started in the JHC. Seventy nine percent of attenders had indicated that they would use a family planning service if it was available. ${ }^{12}$

As we saw similarities between the needs of women attending GUM and family planning services we decided to integrate the two services and in 1992 a FPC, staffed by a female family planning nurse with a female doctor, trained in GUM, HIV and family planning, was introduced to run in conjunction with the WOC. Women have access to free contraceptive advice and a full contraceptive range is available. In addition STD, HIV and serology screening, cervical cytology and sexual health 
counselling are offered. Couples are seen at a separate location within the clinic as requested. An evaluation of the first year is presented.

\section{Method}

A retrospective case note review of women attending the FPC during the first year January-December 1992 was performed. Data were extracted on: prior STDs, parity and pregnancies terminated; screening for STDs, serology and cervical cytology; contraception on first attendance, that supplied, and outcome over the subsequent year 1993.

\section{Results}

One hundred and thirteen women, aged 13-41 years, attended the FPC. Forty five women were new attenders, the remainder were previous $\mathrm{JHC}$ attenders.

Six women were known to be HIV seropositive. Two women were currently using intravenous drugs and five were previous intravenous drug users (IVDU). There was a history of previous STD in $61(54 \%)$ women and a further $13(11.5 \%)$ women gave a previous history of vaginal candidosis or bacterial vaginosis only. Thirty seven $(32.7 \%)$ women had previously had one or more termination(s) of pregnancy (TOP(s)) with 70 TOPs in total and $13(11.5 \%)$ women had previously received oral post coital contraception (PCC). Twenty (17.7\%) women were using no contraception.

Compared with women attending the JHC there were more young clients but, like JHC attenders, over $60 \%$ were in the age range 21-30 years; most originated from the United Kingdom or other European countries $62 \%$ and $12 \%$, respectively, compared with $72 \%$ and $8 \%$ in JHC attenders. In both clinics approximately $7 \%$ were from Australasia, 4\% from the Caribbean, $1 \%$ from Asia and 5\% from Africa; proportionately more North American, Hispanic, Polynesian and Arab women attended the FPC.

Within three months of FPC attendance 89 (78.8\%) women had genital STD screening performed; syphilis, HIV, hepatitis B serology and cervical cytology were performed in 77 $(68.1 \%), 18(15.9 \%), 13(11.5 \%)$ and 62 $(54.9 \%)$ women respectively. Infections identified were similar to those identified over the same time period in women attending the JHC although the prevalence of Chlamydia trachomatis in diagnosed infections was higher in FPC attenders and epidemiological treatment, predominantly due to a sexual partner with non specific urethritis, was commoner in JHC attenders (table 1). However, the women attending the FPC were unselected and were not specifically matched with JHC attenders although they were similar in age and ethnicity. Cytology abnormalities were: inflammatory (2), borderline (3), human papilloma virus (2), mild dyskariosis (3). No positive syphilis serology or new HIV seropositive results were identified. Five women were found to be hepatitis B surface antibody positive; two had no risk factors for acquisition other than unprotected vaginal sexual intercourse in non high risk areas. Eleven women were found to be non-immune and seven were subsequently vaccinated. In the 11 higher risk women who were either IVDU or HIV seropositive three were found to be hepatitis B surface antibody positive, three were found to be non-immune two of whom commenced a vaccination course, five were inadvertently not tested.

Contraception was changed in $60.8 \%$. Most frequently supplied was the combined oral contraceptive pill (COCP) and an increased number of women (15.9\%) adopted a double method of contraception (table 2).

At their first FPC attendance six women required oral PCC and five were already pregnant. Three women, who were of 6-8 weeks gestation, suspected that they were pregnant but the other two women, who were mid cycle, were unaware of their condition. Four of the pregnant women had been using no contraceptive method, one had been using barrier contraception with male condoms. During the year three women conceived; two who used COCP, non compliant; one used a diaphragm, with unclear compliance. Referral for TOP was made as necessary and contraception was supplied to use after the termination; five women were given the COCP and two women were given male condoms. Out of the eight pregnancies seven were terminated.

Over the following year, 1993, 32 women re-attended the FPC and 48 women reattended the JHC, five specifically for family planning. Forty two women $(37 \cdot 2 \%$ of the

Table 1 Infections identified in women attending the $F P C$ and the $\mathcal{F H C}$

\begin{tabular}{lll}
\hline & \multicolumn{2}{l}{ Number of infections diagnosed } \\
\cline { 2 - 3 } & $F P C$ & \multicolumn{1}{l}{$F$ C } \\
Infection & $n=89$ & $n=2596^{*}$ \\
\hline Vaginal candidosis & $33(37 \cdot 1 \%)$ & $906(34 \cdot 9 \%)$ \\
Bacterial vaginosis & $16(18 \cdot 0 \%)$ & $380(14 \cdot 6 \%)$ \\
Genital warts & $16(18 \cdot 0 \%)$ & $438(16 \cdot 9 \%)$ \\
Genital herpes & $9(10 \cdot 1 \%)$ & $320(12 \cdot 3 \%)$ \\
Chlamydia trachomatis & $7(7 \cdot 9 \%)$ & $98(3 \cdot 8 \%)$ \\
Pelvic inflammatory disease & $3(3 \cdot 4 \%)$ & $99(3 \cdot 8 \%)$ \\
Trichomonas vaginalis & $3(3 \cdot 4 \%)$ & $44(1 \cdot 7 \%)$ \\
$N$. Gonorrhoeae & - & $10(0 \cdot 4 \%)$ \\
Syphilis & - & $4(0 \cdot 2 \%)$ \\
Other infections & $2(2 \cdot 2 \%)$ & $271(10.4 \%)$ \\
Epidemiological treatment & $2(10 \cdot 4 \%)$ \\
\hline
\end{tabular}

${ }^{\star} \mathrm{KC} 60$ diagnoses in women attending the John Hunter Clinic (JHC) in 1992.

Table 2 Contraception at the first FPC attendance and the contraception supplied

\begin{tabular}{lll}
\hline Lead method & $\begin{array}{l}\text { At first FPC } \\
\text { attendance }\end{array}$ & $\begin{array}{l}\text { Supplied after } \\
\text { FPC attendance }\end{array}$ \\
\hline Nil & 20 & - \\
Condom (male) & 39 & 13 \\
Femidom (male) or Femidom & - & - \\
Condom (mo & 8 & 13 \\
Diaphragm & 35 & 72 \\
Oral hormonal contraception & - & 7 \\
Injectable progestogen & 4 & 3 \\
Intrauterine contraceptive & & 2 \\
$\quad$ device & 7 & \\
Others & & 16 \\
Double method & 3 & 2 \\
Non barrier + condom (male) & - & \\
Non barrier + Femidom & - &
\end{tabular}


original cohort) were re-supplied with contraception; four required PCC; two intentional pregnancies occurred. Only one of the women requiring referral for TOP during the audit year returned.

\section{Discussion}

An easily accessible FPC integrated with a GUM and HIV clinic provides coordinated sexual health care combining contraceptive provision with an active STD/HIV/cervical cytology screening programme.

The benefits of screening programmes are most clearly illustrated by considering Chlamydia trachomatis infection. Here, the introduction of FPC screening programmes with contact tracing has resulted in reducing the prevalence of the infection. ${ }^{13} 14$ The issue of selective versus universal screening is contentious. However, when the cost of untreated Chlamydia trachomatis infection is considered, there is a significant financial advantage of universal screening over selective or no screening. ${ }^{15}$

When advising on contraception it is important to consider not only contraceptive efficacy but also the protection against infection afforded by condoms in relation to the individual woman and her partner's lifestyle, sexual risk factors and previous or current infection. Balancing all these aspects is particularly important for many of our patients. The use of condoms with a non barrier method, promoted in Holland as the "Double Dutch" method, ${ }^{16}$ was advised for many of our attenders and adopted by $15 \cdot 9 \%$.

Despite providing co-ordinated health care during the first year there were eight pregnancies and seven TOPs. This represents a conception rate of 70.8 per 1000 women and a TOP rate of 61.9 per 1000 women. It is difficult to give accurate Riverside conception or TOP rates for 1992 owing to changes in area boundaries but the figures appear relatively stable over time and are consistently above the national average. In $199140 \%$ of all Riverside conceptions were terminated, the TOP rate was 30.9 per 1000 fertile women, two and a half times the national average. ${ }^{17}$ Our higher FPC conception and TOP rates may be due to the five women who were pregnant at the first FPC attendance, particularly those who were aware of the pregnancy and requested TOP, thereby using the clinic as a referral site. If the five women are excluded from analysis conception and TOP rates of 27.8 and 18.5 per 1000 women respectively are obtained which may be more representative figures.

In order to reduce unwanted pregnancy it is essential to promote earlier access to FPC services and to increase education particularly about post coital contraception for women and clinic staff who may be the first contact point for women at risk. It is also essential to have adequate supplies of contraception material and so we were concerned about the women who failed to return for review. Although they may have attended another FPC or their general practitioner their contra- ceptive supply was unclear. To clarify this and ensure a streamlined service we now formally discuss future sources of contraceptive supply and arrange follow up either in our own FPC, an alternative FPC or with the general practitioner. A recall system has been introduced for patients who fail to attend.

If pregnancy occurs and TOP is requested easy, rapid referral is essential to reduce the pressure on already vulnerable women.

The effect of the changes to improve FPC re-attendance will be audited in due course. Meanwhile we are encouraged that no non intentional pregnancies occurred in our cohort in 1993.

Funding this type of service remains a problem for GUM clinics. However, it is an extremely popular service as many women utilise both GUM and family planning services when they attend and most appreciate the advantage of a one stop service. With the background of the high TOP rate in Riverside we feel that its presence is essential and continue to finance the project with GUM monies. Avoiding unwanted pregnancy remains a universal challenge.

1 Department of Health 1992. The Health of the Nation: a Strategy for Health in England. Cmd 1986. HMSO, London.

2 Eversley RB, Policar M, White V, et al. Self-reported sexually transmitted diseases among family planning clients: ethnic differences in sexual risk behaviour and HIV risk reduction. Ethnicity \& Disease 1993;3:181-8

3 Rosales M, Dominguez V, Bonacho I, Vidal X. Epidemiology of Chlamydia trachomatis in a population attending a family planning centre. Rev Clin Esp 1993; 192:424-7.

4 Ramstedt K, Forssman L, Giesecke J, Granath F. Risk factors for Chlamydia trachomatis infection in 6810 young women attending family planning clinics. Int $f$ STD AIDS 1992;3:117-22.

5 Weinstock HS, Bolan GA, Kohn R, Balladares C, Back A Oliva G. Chlamydia trachomatis infection in women: a need for universal screening in high prevalence populaneed for universal screening in high prov
tions? Am $\mathcal{F}$ Epidemiol 1992;135:41-47.

6 Addiss DG, Vaughn ML, Golubjatnikov R, Pfister J, Kurtycz DFI, Davis JP. Chlamydia trachomatis infection in women attending urban midwestern family planning and community health clinics: risk factors, selective screening and evaluation of non-culture techniques. Sex Transm Dis 1990;17:138-46.

7 Adler MW, Belsey EM, Rogers JS. Sexually transmitted diseases in a defined population of women. BMF 1981; 283:29-32.

8 Riordan T, Macaulay ME, James JM, et al. A prospective study of genital infections in a family-planning clinic. 1 . Microbiological findings and their association with vaginal Mymptoms. Epidemiol Infect 1990;104:47-53.

9 Spence MR, Robbins SM, Polansky M, Schable CA. Seroprevalence of human immunodeficiency virus type 1 (HIV-1) antibodies in a family-planning population. Sex Transm Dis 1991;18:143-5.

10 Asboe D, Boag FC, Evans B. Women's health: potential for better co-ordination of services. Genitourin Med 1992;68:65.

11 Queen HF, Ward H, Smith C, Woodroffe C. Women's health: potential for better co-ordination of services. Genitourin Med 1991;67:215-9.

12 McCarthy GA, Cockell AP, Kell PD, Beevor AS, Boag FC. A women-only clinic for HIV, genitourinary mediFC. A women-only clinic for HIV, genitourinary medi-
cine and substance misuse. Genitourin Med 1992;68: cine and

13 Ripa T. Epidemiological control of genital Chlamydia trachomatis infections. Scand $\mathcal{F}$ Infect Dis, Supp1. 1990; 69:157-67.

14 Addiss DG, Vaughn ML, Ludka D, Pfister J, Davis JP. Decreased prevalence of Chlamydia trachomatis infection associated with a selective screening program in family planning clinics in Wisconsin. Sex Transm Dis 1993; 20:28-35.

15 Humphreys JT, Henneberry JF, Rickard RS, Beebe JL. Cost-benefit analysis of selected screening criteria for Chlamydia trachomatis infection in women attending Colorado family planning clinics. Sex Transm Dis 1992;19:47-53.

16 Nyman V. Going Dutch-a pipe dream? Br f Fam Plan 1993;19:200-203.

17 Riverside Population Health Monitoring 1991. London: Riverside Public Health Department, 1992. 